\title{
An Empirical Study on a Corpus-based Model of Teaching Second Language Writing for English Majors
}

\author{
XIA Jie \\ School of Foreign Languages and Cultures \\ Jiangsu University of Science and Technology \\ Zhenjiang, Jiangsu Province, China \\ xiajie132@163.com
}

\begin{abstract}
This study constructs a corpus-based model of second language teaching for English majors, applying corpus techniques and resources to the teaching of English writing. A comparative empirical study lasting 16 weeks was conducted to testify the efficiency of the new model. Results show that after a 16-week corpus-based instruction the experimental group outperformed the control group in score. In terms of the writing proficiency, the experimental group has made obvious progress in accuracy and complexity of writing while the control group hasn't displayed obvious improvement. Questionnaire and interview are also employed to examine the efficiency of the new model. It is concluded that the new model is conducive to the improvement of second language writing as well as the fostering of autonomous learning ability.
\end{abstract}

Keywords-corpus-based; teaching model; second language writing

\section{INTRODUCTION}

With the advent of corpus technology, corpus-based teaching models have been proposed to improve the current situation of second language teaching in China. Early in the 1990 s, there has been a tendency in the application of corpus in linguistic study. In recent years, researchers in China have analyzed the feasibility of applying corpus in language teaching and have conducted studies on language teaching practice [1][2]. Reference [3] conducted research on the establishment of corpus-based platform for teaching of English writing and worked out several methods for teaching of English writing. Reference [4] constructed an autonomous learning platform for English writing based on internet and corpus technology. Reference [5] formulated a " $3+1$ "teaching model of English writing for non-English majors and conducted an empirical study on the efficiency of the new model writing are insufficient. This study proposes a corpusbased model of teaching English writing for English majors, analyzing the roles that students and teachers play in the whole writing process and exploring the methods of applying corpus technology to teaching English writing. More importantly, a comparative empirical study along with a questionnaire and interview were used to examine the efficiency of the new model and students' opinions about it.
Three research questions are addressed in this study.

(1) Compared with traditional teaching model, can the new corpus-based model improve students' writing score?

(2) Compared with traditional teaching model, is the new corpus-based model conducive to the enhancement of students' writing proficiency?

(3) What are students' opinions on the new model?

However, writing is an output process which hasn't received much attention in second language teaching. In addition, empirical studies on the application of modern information technology to English

\section{A CORPUS-BASED MODEL}

The new model is in line with the current concept to apply corpus technology in English teaching. It is based on two prominent theories in language pedagogy, namely the input and output hypothesis in SLA [6] and scaffolding instruction. It lays equal emphasis on the role of input and output in second language acquisition and integrates modern corpus technology in the teaching of second language writing.

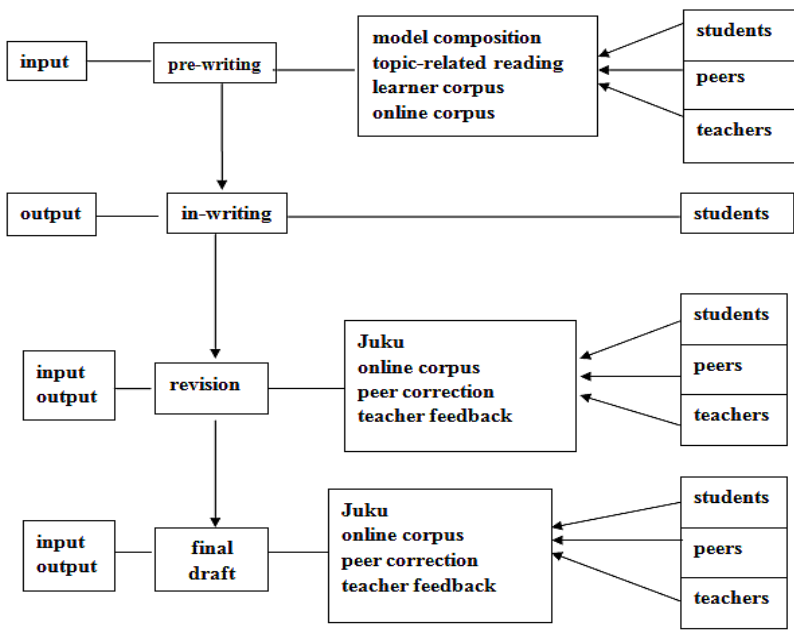

Fig. 1 A corpus-based model of teaching English writing 
As is shown in Fig. 1, the new model divides the writing process into four stages, namely, pre-writing, in-writing, revision and final writing, among which pre-writing involves language input and in-writing involves language output while revision and final writing involve both input and output respectively. The activities that might be involved for each stage are displayed. For the role of teachers and students, only the in-writing stage involves the students solely while other three stages have students, peers and teachers involved.

The new model regards English writing as a process instead of an end, integrating input and output activities alike. Compared with the traditional teaching model, this model is not static in linear order but dynamic in a circulating manner, which displays the interaction between human and machine, teachers and students, as well as students and peers. It encourages students make full use of the corpus resources available on the Internet before the writing stage, and guides through the revision and final writing stages by intelligent use of the online corpus tool Juku and online corpus like COCA and $\mathrm{BNC}$ for correcting and revising compositions autonomously.

\section{Methodology}

\section{A. Research subjects}

Participants are freshmen of two natural classes in Jiangsu University of Science and Technology. One is chosen as the experimental group with 19 students and another as the control group with 20 students. Their writing courses are taught by the same teacher with 5-year teaching experience, familiar with the traditional and new model of teaching English writing. The writing course of the experimental group is taught in accordance with the new corpus-based model and that of the control group is taught based on the traditional model.

\section{B. Research instruments}

Instruments used in this study include two composition materials for assessment in pre-test and post-test. One is picture composition and another is topic composition. In addition, a questionnaire and an interview are employed to examine students' opinions about the new teaching model.

\section{Data collection}

The experiment lasted for a semester. The experimental group follows the new corpus-based teaching model while the control group follows the traditional teaching model. Data are collected in the following aspects.

1) Scores of pre-test and post-test of experimental group and control group

The pre-test score is the average of the scores given by two English teachers according to the CET-4 composition grading criterion. The after-test score is given in the same way as the pre-test.

An independent t-test for pre-test is conducted by SPSS 17.0 to ensure there is no significant difference between the scores of the two groups before the experiment. After the experiment, an independent t-test for post-test is conducted to compare whether there is salient difference in the scores of the two groups after the experiment.

2) Writing Proficiency of experimental group and control group

A paired t-test is conducted by SPSS 17.0 to investigate the changes of writing proficiency of the two groups in pretest and post-test.

The writing proficiency is evaluated in terms of three aspects, including fluency, accuracy and complexity. Fluency is calculated by the total number of words in the composition. The more words it has, the more fluent it is. Accuracy is measured in terms of the percentage of grammatical errors in the composition. Complexity includes syntactic complexity and lexical complexity. Syntactic complexity is measured by the ratio of subordinate clauses to all sentences. Lexical complexity is measured by the ratio of tokens and types [7] calculated by Range 32 .

3) Questionnaire and interview to students in the experimental group

After the experiment, questionnaires are given to students in the experimental group. All 19 questionnaires are accumulated for data analysis. A face-to-face interview is conducted by randomly selecting 5 students in the experimental class, asking questions about their opinions on the new model. Major points in their answers are recorded for further analysis.

\section{RESULTS AND DisCUSSION}

\section{A. Scores of pre-test and post-test of experimental group and control group}

The scores of pre-test and post-score are the average of the two scores given by two English teachers. The Pearson correlation coefficient is 0.672 , and there is significant relevance at $0.01(\mathrm{sig})$ level. The average score of experimental group in pre-test is 9.5789 and that of control group in pretest is 8.9000 with a standard deviation of 0.6789 . Sig. of Levene's test is $0.384(>0.05)$, so 2-tailed sig. is $0.062(>0.05)$, which indicates that there is no significant difference between variables in both groups. Moreover, 0 is included in the $95 \%$ confidence of interval of difference, which also indicates that there is no significant difference in the mean of both groups. Therefore, there is no significant difference in the score s of pre-test and post-test in both groups.

To examine the differences of mean score in post-test of both groups, an independent test for variables in both groups is conducted by SPSS 17.0. The average score of experimental group in post-test is 11.3421 and that of control group in pretest is 9.6250 with a standard deviation of 1.7171 . Sig. of Levene's test is $0.133(>0.05)$, so 2-tailed sig. is $0.000(<0.05)$, which indicates that there is significant difference between variables in both groups. Moreover, 0 is not included in the $95 \%$ confidence of interval of difference, which also indicates that there is significant difference in the mean of both groups. Therefore, there is significant difference in the scores of pretest and post-test in both groups, and the mean score of 
experimental group is remarkably higher than that of the control group.

Statistics above show that the scores of experimental group are greatly improved after practicing the corpus-based model and the new model can improve students' writing performance.

\section{B. Writing Proficiency of experimental group and control group}

To measure the development of the writing proficiency of the experimental group more accurately. We give a statistical analysis of students' writing proficiency in terms of fluency, accuracy and complexity. Paired t-test is conducted by the use of SPSS 17.0 to compare the writing proficiency development of both groups.

It is observed that the 2-tailed sig. of total number of words, total number of errors, type/token ratio and subordinate clauses/sentences ratio are $0.451,0.000,0.001$ and 0.000 respectively. The 2-tailed sig. of total number of words in experimental group is $0.451(>0.05)$, indicating there is no significant difference in the development of fluency in pre-test and post-test of experimental group.

The development of writing proficiency in control group is analyzed in the same way. It is observed that the 2-tailed sig. of total number of words, total number of errors, type/token ratio are higher than 0.05 respectively, indicating there is no significant difference in the development of fluency, accuracy and lexical complexity in pre-test and post-test of control group. The 2-tailed sig. of subordinate clauses/sentences ratio in pre-test and post-test of the control group is $0.002(<0.05)$, showing there is significant difference in syntactic complexity between pre-test and post-test of the control group.

The writing proficiency of the experimental group has been enhanced greatly, mainly in accuracy and complexity and the new corpus-based model can effectively improve the efficiency of teaching English writing.

\section{Students' opinions on the new teaching model}

Results of questionnaire show that $78.95 \%$ students in the experimental group are in favor of the new teaching model. They think that the new teaching model helps them in every step of the writing process and build their confidence in English writing. However, $31.57 \%$ students have problems in the operating skills of corpus tools, thus unable to apply them to English writing.

5 students are randomly chosen as the subjects of the faceto-face structured interview. They think that the new model helps them to improve their writing proficiency and some of them hold that corpus is indispensable in English study. Opinions differ among students of different levels. Students with high scores display intense interest in the new model and are able to apply them in their English writing while students with low scores say that they find it difficult to use corpus to improve English writing.

\section{CONCLUSION}

The study conducted a 16-week empirical study between the new model and the traditional model, together with an investigation on students' opinions on the experimental group. Statistics show that the corpus-based teaching model is more conducive to the improvement of students' scores and writing proficiency, especially in accuracy and complexity. Results in questionnaires and interview indicate that most students think the new model could stimulate their interest in English writing, improve their writing proficiency and foster their ability of autonomous learning. Meanwhile, there are some limitations to this new model, such as the access to computer and internet and the requirements for both teachers and students in the mastery of corpus knowledge and skills. However, the corpusbased teaching model is a promising yet challenging field of study. It is hoped that future study could lay emphasis on an in-depth study of the applications of corpus in English teaching and the effective measures to enhance the teaching efficiency of English writing.

\section{ACKNOWLEDGMENTS}

The paper is supported by the research grants from 2015 Philosophy and Social Science Research Program by Jiangsu Provincial Education Department (2015SJB845), 2015 Social Science Research program by Jiangsu University of Science and Technology (1072921509); 2013 Education and Teaching Reform Program by Jiangsu University of Science and Technology (10704121); 2015 Higher Education Teaching Reform Program by School of Foreign Languages and Cultures, Jiangsu University of Science and Technology.

\section{REFERENCES}

[1] Aanping, He. Corpus linguistics and foreign language teaching [M], Beijing: Foreign Language Teaching and Research Press, 2004

[2] Anping, He. An Introduction to Corpus-assisted English teaching [M], Beijing: Foreign Language Teaching and Research Press, 2010

[3] Jiemin, Pu. A Study on a corpus-based teaching model of English writing [J]. Education and Teaching Research, 2009 (6):70-73.

[4] Hongxue, Dong \& Shenghua, Chu. A study on a corpus-driven teaching model of English writing[J]. Computer-assisted foreign language teaching,2010(3):27-31

[5] Bin, Liu, Lingling, Ge, \& Guangwei, Li, An empirical study on a corpus-based teaching model of English writing[J], Foreign Language and Literature, 2012(4):131-135

[6] M, Swain. Three functions of output in second language learning[M]. Oxford: Oxford University Press, 1995: 125-144

[7] Diana, Larsern-Freeman. The emergency of complexity, fluency, and accuracy in the oral and written production of five Chinese Learners of English [J]. Applied Linguistics, 2006, 27 (4): 590-619 